\title{
Der Einsatz von Pflanzentests bei der Sedimentbewertung
}

\author{
Ute Feiler $\cdot$ Evelyn Claus $\cdot$ Denise Spira $\cdot$ Peter Heininger
}

Eingegangen: 11. März 2009/Akzeptiert: 29. April 2009/Online veröffentlicht: 16. Mai 2009

(C) Springer-Verlag 2009

Zusammenfassung Hintergrund und Ziel Aquatische Organismen der drei Trophiestufen (Produzenten, Konsumenten, Destruenten) werden seit langer Zeit in der ökotoxikologischen Gewässerbewertung eingesetzt (HABAB 2000; HABAK 1999). Typischerweise kommen in bisher verwendeten standardisierten Biotestsystemen Algen, Daphnien und Leuchtbakterien als Testorganismen zum Einsatz. Vergleichende Untersuchungen haben gezeigt, dass die bisher zum Nachweis phytotoxischer Wirkungen herangezogenen Algen nicht für alle Pflanzenschadstoffe hinreichend empfindlich sind und somit als alleinige Vertreter allen autotrophen Lebens nicht ausreichen. Auch in der EG-Wasserrahmenrichtlinie (EU-WFD 2000) werden Makrobenthos, Fische, Phytoplankton und Makrophyten als Indikatoren des Gewässerzustandes gleichberechtigt nebeneinander gestellt. Trotzdem werden Biotestsysteme mit höheren Pflanzen bei der Bewertung von Gewässersedimenten bislang nur wenig eingesetzt. Demgegenüber steht die Entwicklung und Normierung des aquatischen Lemnatests (DIN EN ISO 20079) und die Entwicklung des Sedimentkontakttests mit Myriophyllum aquaticum (Feiler et al. 2004). Letzterer war Bestandteil eines BMBF-Verbundprojekts (SeKT), in dem verschiedene Sedimentkontakttests miteinander vergleichend untersucht wurden (Feiler et al. 2005). Ein Biotest mit Makrophyten hat den Vorteil, dass dessen Resultate leichter auf andere höhere Pflanzen übertragbar sind als Ergebnisse aus einem Algentest. Ziel- und Testorganismus stehen sich entwicklungsbiologisch näher.

Verantwortliche Herausgeber: Jan Schwarzbauer · Peter Heininger · Evelyn Claus

U. Feiler $(\varangle) \cdot$ E. Claus $\cdot$ D. Spira $\cdot$ P. Heininger

Bundesanstalt für Gewässerkunde, Qualitative Gewässerkunde,

Am Mainzer Tor 1, 56068 Koblenz, Deutschland

E-Mail: feiler@bafg.de
In der hier vorgestellten Arbeit wird gezeigt, dass der Einsatz von Biotests mit höheren Pflanzen bei der Analyse von anthropogen belasteten Sedimenten wertvolle Ergebnisse liefert, die in ein Konzept für eine integrierte Gewässerbewertung einfließen können.

Schlüsselwörter Phytotoxizität · Sedimentkontakttest · Pflanzen · Gewässerbewertung · Makrophyt

\section{Aquatic plant bioassays used in the assessment of sediment quality}

Abstract Background and aim Aquatic organisms of the three trophic levels (producers, consumers, reducers) have been used for a long time for ecotoxicological assessments of water quality (HABAB 2000; HABAK 1999). In biotest systems that have become established standards, algae, daphnia, and luminescent bacteria as test organisms are often used so far. However, comparative studies have shown that algae are not sufficiently sensitive to all phytotoxic agents and thus cannot be the exclusive indicator organism of any toxic effects on autotrophic life. Moreover, the EU Water Framework Directive (EU-WFD 2000) sets macrobenthos, fish, phytoplankton, and macrophytes equally side by side as indicators of the status of waters. Nevertheless, biotest systems relying on higher plants have been rarely used in assessments of aquatic sediments so far. Against this background, the aquatic duckweed test (DIN EN ISO 20079) was developed and standardized, and a sediment contact test was developed with Myriophyllum aquaticum (Feiler et al. 2004). The latter was subject of a joint research project of the German Federal Ministry of Education and Research (BMBF-Verbundprojekt "SeKT") that examined and compared several sediment contact tests (Feiler et al. 2005). Compared to tests with algae, 
a biotest using macrophytes has the advantage to be more representative of higher plants, since target organism and test organism are closer related in terms of evolutionary history.

The present study demonstrates that the application of biotests with higher plants in analyses of contaminated sediments yields valuable results that may contribute to a concept for the integrative assessment of water quality.

Keywords Phytotoxicity - Sediment contact assay · Plants $\cdot$ Water quality assessment $\cdot$ Macrophytes

\section{Problemstellung}

Zur Bewertung der Gewässergüte werden seit langer Zeit standardisierte Biotestsysteme mit Algen, Daphnien und Leuchtbakterien als Testorganismen eingesetzt. Es hat sich jedoch gezeigt, dass die Algen als einzige pflanzliche Vertreter in dieser Testpalette nicht ausreichen, um alle von Sedimenten ausgehenden phytotoxischen Wirkungen anzuzeigen. Trotz der mittlerweile erfolgten Entwicklung und Normierung des aquatischen Lemnatests (DIN EN ISO 20079) und der Entwicklung des Sedimentkontakttests mit Myriophyllum aquaticum (Feiler et al. 2004), wurden solche Biotestsysteme bei der Bewertung von Gewässersedimenten bislang nur wenig eingesetzt (Stesevic et al. 2007).

\section{Ziel}

In der hier vorgestellten Arbeit soll gezeigt werden, dass der Einsatz von Biotests mit höheren Pflanzen bei der Untersuchung von anthropogen belasteten Sedimenten wertvolle Ergebnisse liefert, die in ein Konzept für eine integrierte Gewässerbewertung einfließen können.

\section{Ergebnisse und Diskussion}

Um die Eignung von Pflanzentests für die Untersuchung von Gewässersedimenten nachzuweisen, wurden beispielhaft ausgewählte, unterschiedlich anthropogen belastete und gering belastete, natürliche Sedimente aus mehreren Flüssen (Tabelle 1) mit zwei Pflanzentests sowohl in aquatischen Testansätzen als Porenwasser oder Sedimentextrakte (aquatischer Lemnatest, DIN EN ISO 20079) als auch in Gesamtsedimentansätzen (Myriophyllum-Sedimentkontakttest, Feiler et al., 2004) vergleichend untersucht.

In den Porenwasseruntersuchungen mit dem aquatischen Lemnatest wurden signifikante Unterschiede im Wachstumsverhalten der Lemna minor in Abhängigkeit vom untersuchten Sediment beobachtet. In Abb. 1 (schwarze Säulen) sind die Wachstumsänderungen von L. minor in der jeweils höchs-
Tabelle 1 Ausgewählte Sedimententnahmestandorte und deren prioritäre Belastung

\begin{tabular}{lll}
\hline Fluss & Probennahmestelle & Prioritäre Belastung \\
\hline Mosel & Trier (T) & Gering \\
Mosel & Trier-Monaise (TM) & Mittel \\
Saar & Wadgassen (W) & Hoch \\
Rhein & Ehrenbreitstein (EB) & Gering \\
Elbe & Magdeburg (MA) & Hoch \\
MEW* & Dömitz (DÖ) & Gering \\
Oder & Hohenwutzen (HW) & Mittel \\
\hline
\end{tabular}

* MEW $=$ Müritz-Elde-Wasserstraße

ten Porenwasserkonzentration (Originalansatz) dargestellt. Zugleich wurden die pT-Werte nach Krebs (2000) ermittelt: In den Sedimenten von Magdeburg (MA) pT 5, in Hohenwutzen (HW) pT 4, in Wadgassen (W), Trier (T) und Ehrenbreitstein (EB) jeweils pT 2, und Dömitz (DÖ) und TrierMonaise (TM) pT 0. Ähnliche pT-Werte wurden von Feiler et al. (2006a) für vergleichbare Sedimentproben beschrieben. Nach dem Bewertungsschema von Krebs (2000) zeigen die pT-Werte 5 und 6 eine hohe toxische Belastung der Sedimente an, pT 3 und 4 stehen für eine mittlere, pT 1 und 2 für eine geringe und pT 0 für eine nicht nachweisbare Toxizität.

Die gleichen Sedimentproben wurden auch im Sedimentkontakttest mit Myriophyllum aquaticum untersucht (Abb. 1, weiße Säulen). Hier wurden ebenfalls Wachstumshemmungen und -förderungen von M. aquaticum in Abhängigkeit vom untersuchten Sediment festgestellt. Allerdings reagierten die beiden Pflanzenarten zum Teil unterschiedlich auf den untersuchten Sedimenten. Große Unterschiede wurden z. B. in den Sedimenten der Mosel (T und TM), der Elbe (MA) und der Oder (HW) beobachtet. Ähnliche Reaktionen der beiden Pflanzenarten riefen die Sedimente des Rheins (EB) und der Saar (W) hervor, wobei das Wadgassen-Sediment $(\mathrm{W})$ in beiden Fällen starke Toxizitäten und das Ehrenbreitstein-Sediment (EB) jeweils keine signifikanten Hemmungen hervorriefen (Abb. 1). Beide Pflanzen sind also in der Lage, Phytotoxizität, ausgehend vom Sediment, anzuzeigen und sich dabei in ihrer Aussage zu ergänzen. Die Ursache für diese unterschiedlichen Aussagen könnte in den unterschiedlichen Lebensweisen der Pflanzen liegen: Lemna minor schwimmt auf der Wasseroberfläche und reagiert auf in Porenwasser gelöste Substanzen. Myriophyllum aquaticum dagegen wurzelt im Sediment und steht somit mit den sedimentgebundenen Substanzen in direktem Kontakt. Durch Milieuänderungen um die Wurzel (bedingt durch Pflanzenexsudate) können weitere Substanzen aus dem Sediment für Myriophyllum verfügbar gemacht werden und zu Schadreaktionen führen.

Um Ursachen für die phytotoxischen Wirkungen zu finden, wurden in einem weiteren Ansatz Sedimente extrahiert und 
Abb. 1 Wachstumsvergleich von Lemna minor (Porenwasseransatz) und Myriophyllum aquaticum (Gesamtsedimentansatz) auf verschieden anthropogen belasteten, natürlichen Sedimenten. L\%, M\%: Wachstumshemmung Lemna minor (L) und Myriophyllum aquaticum (M); Sedimentabkürzungen s. Tabelle 1

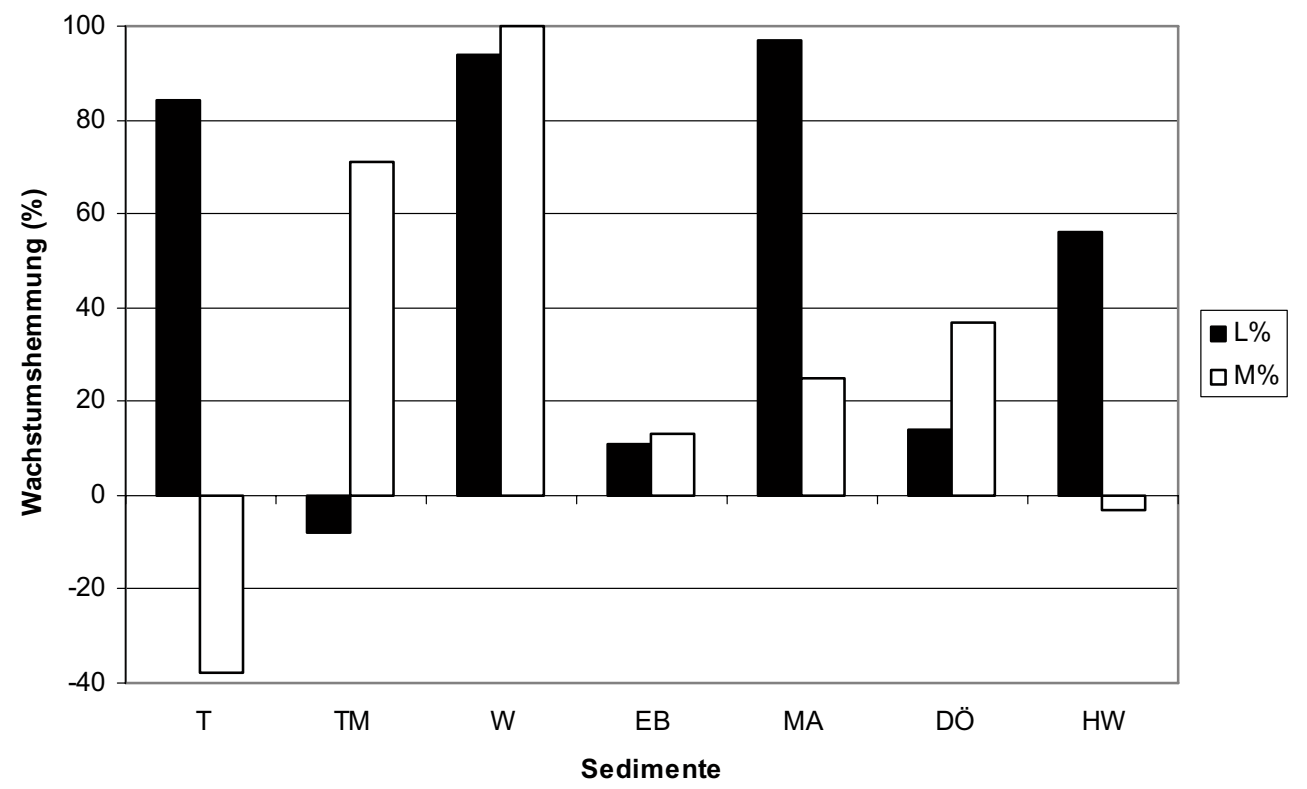

sowohl das Gesamtextrakt als auch Extraktfraktionen mit dem aquatischen Lemnatest untersucht. Die Ergebnisse wurden von Feiler et al. (2002) beschrieben. Es konnte in Extrakten einer Sedimentprobe aus der Elbe (Standort MA) beobachtet werden, dass Lemna minor selektiv Schadstoffe aus den polaren organischen Fraktionen anzeigt. Identifiziert wurden Herbizide (Lenacil, Prometryn, Tridemorph), das Antiseptikum Chorofen, Bisphenol A, Antioxidatien und Alkylphenole. Es ist anzunehmen, dass die in dieser Fraktion enthaltenen Herbizide für die Lemnatoxizität verantwortlich waren.

Mit Myriophyllum aquaticum sind Untersuchungen mit Sedimentextrakten nicht möglich. Es wurde jedoch versucht, die Daten der chemischen Analyse der Sedimente auf gewässertypische, darunter prioritäre, Schadstoffe mit dem Wachstumsverhalten von Myriophyllum aquaticum zu korrelieren. Ein deutlicher Zusammenhang wurde dabei zwischen dem Chromgehalt der Sedimente, speziell der ansonsten eher gering belasteten Probe aus Dömitz/MEW und der Wachstumshemmung von Myriophyllum aquaticum, beobachtet (Feiler et al. 2006b). Ein ähnlicher Zusammenhang wurde auch von Stesevic et al. (2007) mit Sedimenten des Skadar-Sees beobachtet.

\section{Ausblick}

Durch die hier vorgestellten Anwendungsbeispiele für zwei Pflanzentests mit höheren Wasserpflanzen konnte gezeigt werden, dass sich diese Tests für Sedimentuntersuchungen zur ökotoxikologischen Gewässerbewertung eignen und eine wertvolle Ergänzung zum häufig eingesetzten Algentest darstellen. In einer Vergleichsstudie des Algen- und Lemnatests wurden deutliche Unterschiede zwischen den Ergebnissen mit den beiden Testsystemen beschrieben (Feiler et al. 2006a).
Wirkungsorientierte Untersuchungen von Sedimentextrakten, ausgehend von den Ergebnissen in Pflanzentests, können einen nützlichen Beitrag zur Gewässerbewertung leisten.

\section{Literatur}

DIN EN ISO 20079 (2006) Water quality - Determination of the toxic effect of water constituents and waste water to duckweed (Lemna minor) - Duckweed growth inhibition test

EU-WFD (2000) European Union Water Framework Directive 2000/60/EG of the European Parliament. J Eur Community

Feiler U, Claus E, Heiniger P (2002) Der Einsatz von Pflanzentests bei der Sedimentbewertung. Ummweltwiss Schadst Forsch 14:8-11

Feiler U, Kirchesch I, Heininger P (2004) A new plant bioassay for aquatic sediments. J Soils Sediments 4:261-266

Feiler U, Ahlf W, Höss S, Hollert H, Neumann-Hensel H, Meller M, Weber J, Heininger P (2005) The SeKT Joint Research Projekt: definition of reference conditions, control sediments and toxicity thresholds for limnic sediment contact tests. Environ Sci Pollut Res 12:257-258

Feiler U, Krebs F, Heininger P (2006a) Aquatic plant bioassays used in the assessment of water quality in German rivers. Hydrobiologia 570:67-71

Feiler U, Pelzer J, Heininger P (2006b) Entwicklung und Erprobung eines Sedimentkontakttests mit höheren Pflanzen zur Beurteilung der Gewässerqualität. BfG-1491, German Federal Institute of Hydrology (BfG), Koblenz

HABAK-WSV (1999) Handlungsanweisungen für den Umgang mit Baggergut im Küstenbereich. BfG-1100, Koblenz

HABAB-WSV (2000) Handlungsanweisungen für den Umgang mit Baggergut im Binnenland. BfG-1251, Koblenz

Krebs F (2000) Ökotoxikologische Bewertung von Baggergut aus Bundeswasserstraßen mit Hilfe der pT-Wert-Methode. Hydrologie Wasserbewirtsch 44:301-307

Stesevic D, Feiler U, Sundic D, Mijovic S, Erdinger L, Seiler TB, Heininger P, Hollert H (2007) Application of a new sediment contact test with Myriophyllum aquaticum and of the aquatic lemna test to assess the sediment quality of Lake Skadar. J Soils Sediments 7:342-349 